\title{
In prostatic transition zone lesions (PI-RADS v2.1): which subgroup should be biopsied?
}

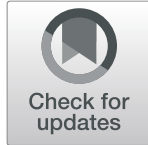

Jihae An¹, Young Joong Kim¹, Jae Young Seo ${ }^{1}$, Cheol Mog Hwang ${ }^{1}$, Dong Hyun Oh¹, Mu-Sik Lee ${ }^{2}$ and Keum Won Kim ${ }^{1 *}$ (iD

\begin{abstract}
Background: The study aimed to compare the diagnostic performance of T2-weighted imaging (T2WI) score 3 transition zone (TZ) lesions between Prostate Imaging and Reporting Data System (PI-RADS) V2.1 and modified PIRADS v2.1-B.

Results: Among TZ lesions ( $n=78), 47(60.0 \%)$ had T2WI score of 3, and 16 of the 47 (34.0\%) were malignant. The rate of malignancy was $8.8 \%$ in PI-RADS category $3 \mathrm{~A}, 100 \%$ in PI-RADS category $3 \mathrm{~B}$, and $100 \%$ in PI-RADS category 4. The apparent diffusion coefficient value of PI-RADS category $3 B\left(0.934 \pm 0.158 \times 10^{-3} \mathrm{~mm}^{2} / \mathrm{s}\right)$ showed significant difference with that of PI-RADS category 3A $\left(1.098 \pm 0.146 \times 10^{-3} \mathrm{~mm}^{2} / \mathrm{s}\right)$ but none with PI-RADS category $4(0.821$ $\left.\pm 0.091 \times 10^{-3} \mathrm{~mm}^{2} / \mathrm{s}\right)$. There was no significant difference in the sensitivity and negative predictive value of PIRADS V2.1 and PI-RADS V2.1-B. Specificity and positive predictive value of modified PI-RADS V2.1-B were much higher than those of PI-RADS v2.1 for both readers $(p<.001)$. The area under the receiver operating characteristic curve tended to be higher with PI-RADS v2.1-B than with PI-RADS v2.1.
\end{abstract}

Conclusion: Biopsy for PI-RADS 3B lesion is necessary due to its superior malignancy potential than that of PI-RADS 3A lesion.

Keywords: Multiparametric magnetic resonance imaging, Prostate cancer, Prostate Imaging and Reporting Data System

\section{Background}

Prostate cancer is the second most frequently diagnosed cancer and the fifth leading cause of cancer mortality among men worldwide $[1,2]$. The incidence of prostate cancer has increased since the 1990s due to the implementation of prostate-specific antigen (PSA) testing and development of a cancer registration system [3]. The incidence varies greatly across the globe and has been increasing in most Asian countries [3, 4]. Despite the majority of prostate cancer occurring in the peripheral zone (PZ), up to $30 \%$ of prostate cancer occurs in the transition zone (TZ) [5].

\footnotetext{
* Correspondence: radkim14@gmail.com

${ }^{1}$ Department of Radiology, Konyang University Hospital, Konyang Univ.

Hospital 158, Gwanjeodong-ro, Seo-gu, Daejeon, Korea

Full list of author information is available at the end of the article
}

Among the various diagnostic tools available, multiparametric magnetic resonance imaging (mpMRI) has been widely used for prostate-cancer detection and risk stratification. According to a recent meta-analysis, the sensitivity and specificity of mpMRI reached 0.87 and 0.68 , respectively, and especially accessing highly malignant lesion, mpMRI had better diagnostic accuracy compared to biparametric MRI [6, 7].. The application of mpMRI for diagnosing prostate cancer has been recommended in the National Comprehensive Cancer Network, European Association of Urology, and European Society of Urogenital Radiology guidelines [8].

The American College of Radiology, European Radiology of Uroradiology, and AdMeTech Foundation jointly put forth the Prostate Imaging and Reporting Data System (PI-RADS) Version 2 in 2015 to standardize the assessment of the probability of clinically significant 
prostate cancer using mpMRI. However, due to limitations such as suboptimal inter-reader responsibility, a high false-negative rate for the lower PI-RADS assessment category, and a lower detection rate for $\mathrm{TZ}$ prostate cancer (TZPC) than for PZ prostate cancer, the updated PI-RADS Version 2.1 (PI-RADS v2.1) was developed in 2019 [9-11]. Several studies suggested that PI-RADS v2.1 is preferable for evaluating transition-zone lesions and showed that it provided comparable interreader agreement [12-14]. One study reported that typical benign prostatic nodules in the TZ were downgraded, but there were no significant changes in the number of positive and negative MRI results identified using PI-RADS v2.1 [15]. This means that PI-RADS v2.1 is expected to have little influence on clinical management.

Although biopsy should be considered for PI-RADS 4 or 5 (but not for PI-RADS 1 or 2), PI-RADS v2.1 does not include recommendations for management. This is because factors other than MRI findings must be taken into account, including clinical history, laboratory findings, local preferences, expertise, and standards of care. Thus, biopsy may or may not be appropriate for PIRADS 3, depending on these other factors. One of the major modifications in the updated version 2.1 is the diagnostic criteria for TZPC on T2-weighted images. The following were considered PI-RADS 3 lesions: lesions with a T2WI score of 3 and DWI score of 4 or less, T2WI score of 2, and DWI score of 4 or more. Should all PI-RADS 3 lesions in the TZ be biopsied? Our findings shed light on this question.

This study aimed to compare the diagnostic performances of PI-RADS v2.1 and PI-RADS v2.1-B in detecting TZPC and propose the sub-classification of PI-RADS category 3 lesions for a more accurate evaluation that could improve clinical treatment.

\section{Methods}

Our institutional review board approved this study, and the need to obtain informed patient consent was waived due to the retrospective nature of the study.

\section{Patient population}

This study included 80 patients with pathologically proven prostate cancer at our institution between March 2014 and February 2019 were included; their age ranged from 59 to 83 years, and mean age \pm SD was $70.7 \pm 5.82$ years.

\section{Inclusion criteria}

Patients who underwent mpMRI prior to radical prostatectomy were included.

\section{Exclusion criteria}

Patients with low-quality mpMRI image; patients with incomplete investigation of clinicopathologic factors; and patients who were treated with neoadjuvant chemotherapy before surgery were excluded (Fig. 1).

\section{Clinicopathologic information}

Data on preoperative serum PSA, total cancer size, Gleason score (GS), seminal-vesicle invasion, lymphnode invasion, extracapsular extension, and tumor location were retrospectively collected. The GSs were evaluated according to the 2014 International Society of Urological Pathology Modified Gleason Grading System [16] and divided into three groups: GS 6, GS 7, and GS 8-9. The presence of seminal-vesicle invasion, lymph-node invasion, and extracapsular extension was investigated (Table 1).

\section{MR image technique}

MR examinations were performed with Achieva 3.0T MRI (Philips Medical Systems, Netherlands) and Ingenia 3.0T CX (Philips Medical Systems, Netherlands) using a pelvic phased-array coil. All images were taken with the patients in the supine position. After acquisition of the localizing image by sagittal T2-weighted turbo spin-echo (TSE) imaging, an adequate scan range was established.

Axial, coronal, and sagittal T2-weighted TSE images of the prostate and seminal vesicles were obtained with the following parameters: repetition time/echo time/fractional anisotropy (TR/TE/FA) (ms) 3184.6/ $140 / 90$, field of view $(F O V) 180 \times 180$, section thickness $3 \mathrm{~mm}$, intersection gap $1 \mathrm{~mm}$, matrix size $320 \times$ 320 , and number of excitations (NEX) 2. T2-weighted fat-saturation axial images were investigated using the following parameters: TR/TE/FA (ms) 2729.5/70/90, matrix size $320 \times 257$, and other parameters with T2weighted TSE images. Axial T1-weighted TSE images were obtained using TR/TE/FA (ms) 530.9/10/90, FOV $180 \times 180$, section thickness $3 \mathrm{~mm}$, intersection gap $1 \mathrm{~mm}$, matrix size $320 \times 320$, and NEX 2. Axial diffusion-weighted imaging (DWI) of the prostate was performed using a single-shot echoplanar imaging technique and a $b$ value of $1000 \mathrm{~mm}^{2} / \mathrm{s}$ with the following parameters: TR/TE/FA (ms) 6000/69.5/90, FOV $180 \times 180$, section thickness $3 \mathrm{~mm}$, intersection gap $0 \mathrm{~mm}$, matrix size $72 \times 71$, and NEX 7 . ADC values were obtained from DWI sequences performed with $b$ values of 0 and $1000 \mathrm{~mm}^{2} / \mathrm{s}$. Dynamic contrast-enhanced (DCE) images were obtained with TR/TE/FA 3.3/1.6/10, FOV $200 \times 200$, section thickness $6 \mathrm{~mm}$, intersection gap $0.5 \mathrm{~mm}$, matrix size 200 $\times 200$, and NEX 4 (Table 2). 


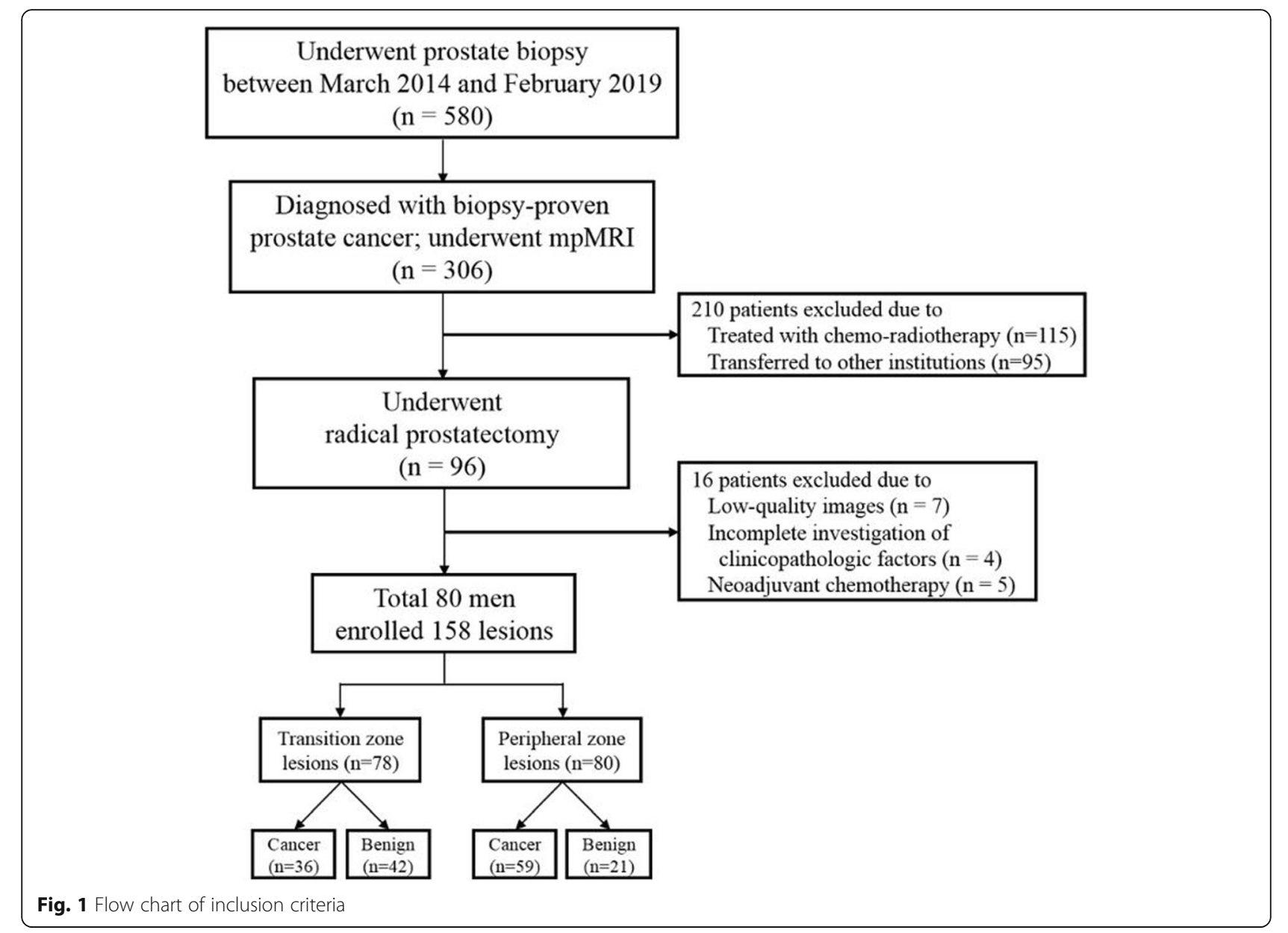

\section{MR image analysis}

A uroradiologist with more than 15 years of experience in interpreting MR prostate examinations (reader 1) and a radiologist with 3 years of clinical experience (reader 2) reviewed the mpMRIs. First, the detection and interpretation of prostate cancer were based on PI-RADS v2.1; prostate cancers in the PZ were assessed with DWI as the dominant sequence, and TZPC with a T2WI sequence. Apparent diffusion coefficient (ADC) values were obtained from the region of interest (ROI) of the center of the detective lesion. ROI sizes were selected as either the smallest or the default size allowed by the PACS workstation (INFINITT PACS M6, Infinitt Healthcare Co., Seoul, Korea).

Then, all lesions were reanalyzed using modified PIRADS v2.1-B with the new PI-RADS category subclassification. We subclassified PI-RADS category 3 TZ lesions as PI-RADS category 3B (biopsy-needed lesion; the lesion revealing T2WI score 3 with DWI score 4) and PI-RADS category 3A (no biopsy-needed lesion; the lesion revealing T2WI score 3 with DWI scores 1 , 2, and 3) (Fig. 2). Two radiologists independently assessed the lesions in the $\mathrm{TZ}$ and $\mathrm{PZ}$ of the prostate gland and scored them from 1 to 5 for T2WI and DWI using PIRADS v2.1 and modified PI-RADS v2.1-B, respectively. In case of disagreement, the final decision was made by an experienced uroradiologist.

\section{Statistical analysis}

All statistical analyses were performed using Statistical Package for the Social Sciences (SPSS) version 20.0 (SPSS Inc., Chicago, IL, USA).

To evaluate interobserver agreement for the categorization of lesions according to PI-RADS v2.1 and modified PI-RADS v2.1-B, we used receiver operating characteristic curve analysis and compared the areas under the receiver operating characteristic curve between two readers. We also computed the weighted quadratic kappa coefficients. After consensus (in case of disagreement), the diagnostic performances for TZPC of PI-RADS v2.1 and PI-RADS v2.1-B were evaluated using the chi-square test. Chi-square and independent sample $t$ tests were used to compare ADC values and clinicopathologic characteristics between prostate lesions. The level of statistical significance was set at $p<0.05$. 
Table 1 Baseline characteristics of enrolled patients with pathologically proved prostate cancer

\begin{tabular}{|c|c|}
\hline Clinicopathologic findings & Patients $(n=80)$ \\
\hline Age (years; mean, range) & $70.7(59-83)$ \\
\hline \multicolumn{2}{|l|}{ Serum PSA } \\
\hline Total PSA & $13.09(1.3-66.2)$ \\
\hline Free PSA & $1.70(0.2-7.2)$ \\
\hline \multicolumn{2}{|l|}{ Cancer location } \\
\hline Transition zone & 22 \\
\hline Peripheral zone & 44 \\
\hline Transition and peripheral zone & 14 \\
\hline \multicolumn{2}{|l|}{ Pathologic T stage } \\
\hline $\mathrm{T} 2 \mathrm{a}, \mathrm{T} 2 \mathrm{~b}$ & 16 \\
\hline $\mathrm{T} 2 \mathrm{C}$ & 41 \\
\hline Т3а & 13 \\
\hline T3b & 10 \\
\hline \multicolumn{2}{|l|}{ Pathologic N stage } \\
\hline No & 76 \\
\hline N1 & 4 \\
\hline \multicolumn{2}{|l|}{ Seminal vesicle invasion } \\
\hline No & 71 \\
\hline Yes & 9 \\
\hline \multicolumn{2}{|l|}{ Extracapsular extension } \\
\hline No & 49 \\
\hline Yes & 31 \\
\hline \multicolumn{2}{|l|}{ Gleason score (GS) } \\
\hline 6 & 6 \\
\hline 7 & 63 \\
\hline $8-9$ & 11 \\
\hline
\end{tabular}

Table 2 Protocols of each sequence of prostate MRI

\begin{tabular}{llll}
\hline & DW-SENSE & T2WI TSE & DCE-MRI \\
\hline TR/TE/FA (ms) & $6000 / 69.5 / 90$ & $3184.6 / 140 / 90$ & $3.3 / 1.6 / 10$ \\
Matrix size & $72 \times 71$ & $320 \times 320$ & $200 \times 200$ \\
FOV & $180 \times 180$ & $180 \times 180$ & $200 \times 200$ \\
NEX & 7.0 & 2.0 & 4.0 \\
SENSE & 2.0 & 2.0 & 1.5 \\
Thickness (mm) & 3.0 & 3.0 & 6.0 \\
Section gap & 0 & 1.0 & 0.5 \\
Imaging time & $5 \mathrm{~m} \mathrm{06} \mathrm{s}$ & $2 \mathrm{~m} \mathrm{20} \mathrm{s}$ & $5 \mathrm{~m} \mathrm{15} \mathrm{s}$ \\
\hline
\end{tabular}

DW-SENSE diffusion-weighted single-shot echo-planar imaging with sensitivity encoding, DCE-MRI dynamic contrast enhance-magnetic resonance imaging

\section{Results}

This study included patients who underwent mpMRI prior to radical prostatectomy. Ninety-six men were initially included, and 16 of them were excluded. Total 80 patients were included in this study. A total of 158 lesions were detected in 80 patients. Of the 80 patients, 95 of malignant lesions (36 with TZPC, 59 with PZ prostate cancer), and 63 of benign lesions (42 with TZ, 21 with PZ) were detected. Among the TZ lesions $(n=78), 12$ (15.4\%) had T2WI score of 2, $47(60.0 \%)$ had T2WI scores of 3, $7(9.0 \%)$ of T2WI score 4, and $12(15.3 \%)$ of T2WI score 5. The malignancy rate of each T2WI score lesions were $0 \%$ in T2WI score $2,34.0 \%$ in T2WI score 3 , and $100 \%$ in both T2WI score 4 and 5 .

\section{Assessment of T2WI score 3 lesions of TZPC}

In evaluating T2WI score 3 lesions of TZPC, the two readers frequently appeared discordant. For this reason, we compared clinicopathologic characteristics and ADC values between T2WI score 3 lesions of TZPC (Table 3). We subcategorized T2WI score 3 lesions by DWI score: $1 / 2 / 3,4$, or 5 lesions. Among 47 lesions that were evaluated as T2WI score 3,10 were categorized as PI-RADS category $3 \mathrm{~B}$, showing high signal intensity on DWI images $(b=1000)$ at the corresponding nodule with low signal intensity on the ADC map, resulting in a DWI score of 4 . These lesions were categorized as 3 according to PI-RADS v2.1 but as 3B according to PI-RADS v2.1B. All of the lesions in the PI-RADS 3B group were pathologically confirmed as malignant (GS 7) (Fig. 3). Two of the 10 lesions showed extracapsular extension. The average ADC value was $0.934 ; 34$ of the 47 lesions with a T2WI score of 3 were categorized as PIRADS category 3A, showing subtle iso-signal intensity on the DWI image $(b=1000)$ at the corresponding nodule and low signal intensity on the ADC map, resulting in a DWI score of 3 . These lesions were categorized as 3 according to PI-RADS v2.1 but as 3A according to PI-RADS v2.1-B. Thirty-one of these 34 lesions were histologically confirmed as benign hyperplastic nodules (Fig. 4); three of them showed histologically confirmed malignancy (one lesion of GS 6, two lesions of GS 7). These were false-negative cases; we categorized them with a T2 score of 3 and DWI score of 3 , resulting in a final score of 3 based on $\mathrm{v} 2.1$ and $3 \mathrm{~A}$ based on v2.1-B. The patients underwent radical prostatectomy and were confirmed to have TZPC (Fig. 5). The average ADC value was 1.166. Three lesions were included in PI-RADS category 4, as they were more than $1.5 \mathrm{~cm}$ with high signal intensity on the DWI images $(b=1000)$ and low signal intensity on the ADC map, resulting in a DWI score of 5 . These lesions were categorized as 4 according to both PI-RADS v2.1 and PI-RADS v2.1-B. All of the 


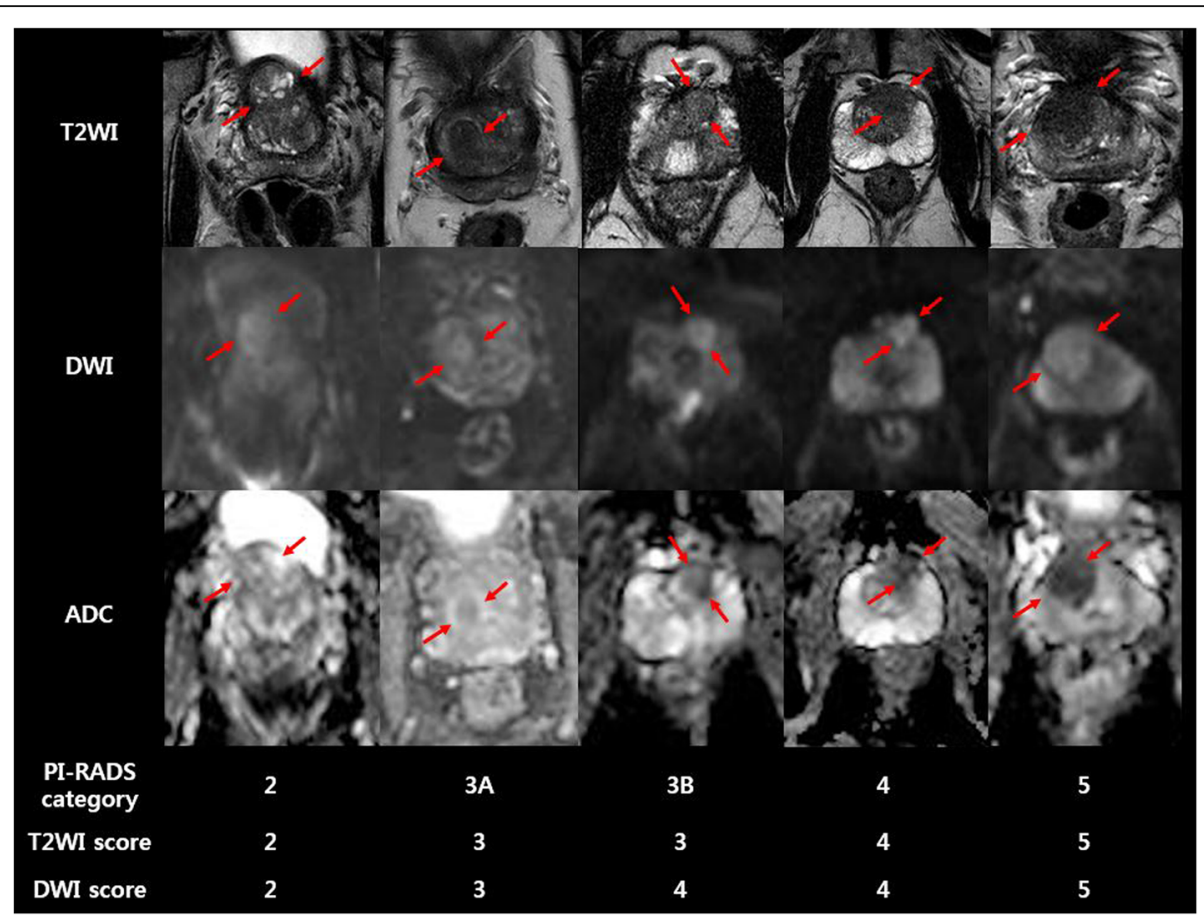

Fig. 2 Examples of transition zone prostate cancer for reader interpreted according to modified PI-RADS V2.1-B. a T2-weighted image (T2WI) showed 17-mm-sized encapsulated nodule with mild hyperintensity (T2WI score 2) in the right transition zone with linear diffusion restriction (DWI score 2). The lesion was categorized as 2 according to PI-RADS V2.1-B. b T2WI showed 12-mm-sized obscured marginated, heterogeneous nodule (T2WI score 3) in the rignt transition zone with mild diffusion restriction (DWI score 3), which was categorized as 3A according to PI-RADS V2.1-B. c T2Wl showed 10-mm-sized obscured, heterogeneous nodule (T2WI score 3) in the left transition zone, with focal marked diffusion restriction (DWI score 4), suggesting category 3B according to PI-RADS V2.1-B. d T2WI showed 13-mm-sized non-circumscribed, homogeneous nodule (T2WI score 4) in the right transition zone, with marked diffusion restriction (DWI score 4). The lesion was categorized as PI-RADS 4. e T2Wl showed $17 \mathrm{~mm}$ sized homogeneous nodule with extracapsular extension in the right transition zone (T2Wl score 5), with marked diffusion restriction (DWI score 5), suggesting PI-RADS category 5

Table 3 Comparison of clinicopathologic characteristics and ADC value between T2WI scores in transition zone prostate cancer

\begin{tabular}{|c|c|c|c|c|c|c|c|}
\hline \multirow[b]{3}{*}{$\begin{array}{l}\text { Modified PI-RADS } \\
\text { category }\end{array}$} & \multirow{2}{*}{$\begin{array}{l}\text { T2WI score } 2(n= \\
12) \\
\text { DWI } 1,2,3\end{array}$} & \multicolumn{3}{|c|}{ T2WI score $3(n=47)$} & \multicolumn{2}{|c|}{ T2WI score $4(n=7)$} & \multirow{2}{*}{$\begin{array}{l}\text { T2WI score } 5(n= \\
12) \\
\text { DWI } 5\end{array}$} \\
\hline & & DWI 1, 2, 3 & DWI 4 & DWI 5 & $\begin{array}{l}\text { DWI 1, 2, } \\
3\end{array}$ & DWI 4 & \\
\hline & Category 2 & $\begin{array}{l}\text { Category } \\
3 A\end{array}$ & $\begin{array}{l}\text { Category } \\
3 B\end{array}$ & Category 4 & $\begin{array}{l}\text { Category } \\
4\end{array}$ & Category 4 & Category 5 \\
\hline Lesion number & 12 & 34 & 10 & 3 & 1 & 6 & 12 \\
\hline \multicolumn{8}{|l|}{ Characteristics } \\
\hline Benign & 12 & 31 & 0 & 0 & 0 & 0 & 0 \\
\hline Malignant & 0 & 3 & 10 & 3 & 1 & 6 & 12 \\
\hline \multicolumn{8}{|c|}{ Extracapsular extension } \\
\hline Positive & 0 & 0 & 2 & 3 & 0 & 1 & 11 \\
\hline Negative & 12 & 34 & 8 & 0 & 1 & 5 & 1 \\
\hline \multicolumn{8}{|l|}{ Gleason score } \\
\hline 6 & 0 & 1 & 0 & 0 & 0 & 0 & 0 \\
\hline 7 & 0 & 2 & 10 & 3 & 0 & 5 & 9 \\
\hline $8-9$ & 0 & 0 & 0 & 0 & 1 & 1 & 3 \\
\hline ADC value & $1.147 \pm 0.140$ & $\begin{array}{l}1.098 \pm \\
0.146\end{array}$ & $\begin{array}{l}0.934 \pm \\
0.158\end{array}$ & $\begin{array}{l}0.821 \pm \\
0.091\end{array}$ & 1.001 & $\begin{array}{l}0.874 \pm \\
0.126\end{array}$ & $0.697 \pm 0.122$ \\
\hline
\end{tabular}



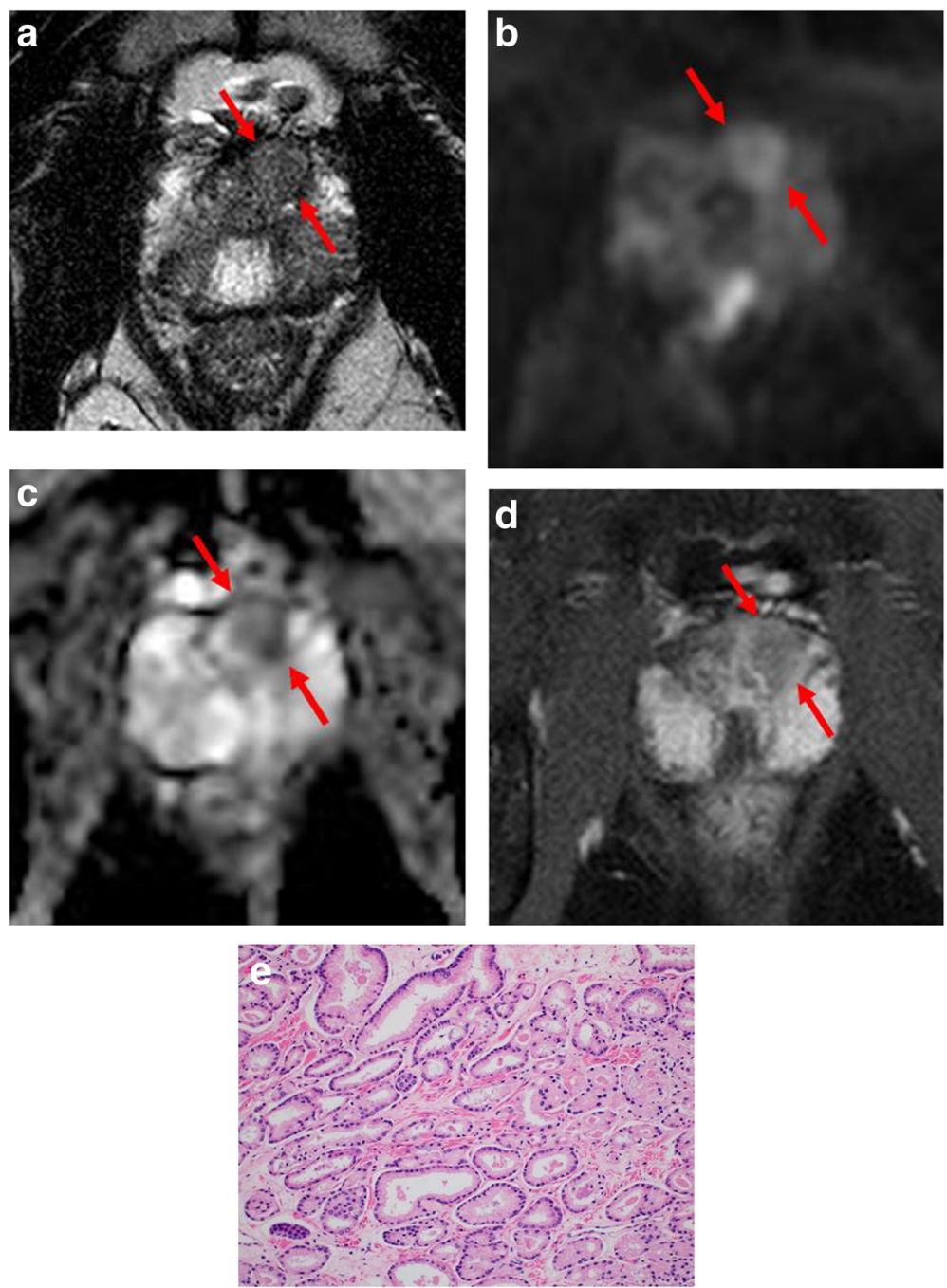

Fig. 3 Multiparametric magnetic resonance image of a 60-year-old patient who underwent radical prostatectomy (serum prostate-specific antigen $4.1 \mathrm{ng} / \mathrm{ml}$, apparent diffusion coefficient [ADC] 0.887). An axial T2-weighted image (T2WI) (a) and dynamic contrast-enhanced image (b) indicate an approximately $1 \mathrm{~cm}$, heterogeneous, obscured, marginated nodule with minimal enhancement in the left transition zone (arrows), resulting in a T2WI score of 3. Diffusion-weighted imaging (DWI) $(b=1000)$ (c) demonstrated high signal intensity at the corresponding nodule with low signal intensity on an ADC map (d), resulting in a DWI score of 4. This lesion was categorized as 3 according to PI-RADS V2.1 but as 3B according to PI-RADS v2.1-B. The patient underwent radical prostatectomy, which confirmed transition-zone cancer with a Gleason score of 7 (4 $+3)$. The procedure revealed multiple poorly formed glands with peripherally arranged nuclei and a macronucleolus (e)

lesions in the PI-RADS 4 group showed extracapsular extension, and histologically confirmed malignancy (GS 7). The average ADC value was 0.821 . The ADC value of PI-RADS category $3 \mathrm{~B}\left(0.934 \pm 0.158 \times 10^{-3}\right.$ $\mathrm{mm}^{2} / \mathrm{s}$ ) differed significantly with that of PI-RADS category 3A $\left(1.098 \pm 0.146 \times 10^{-3} \mathrm{~mm}^{2} / \mathrm{s}\right)$ but showed no difference when compared with PI-RADS category 4 $\left(0.821 \pm 0.091 \times 10^{-3} \mathrm{~mm}^{2} / \mathrm{s}\right)$. The malignancy rate of T2WI score 3 lesions was $34.0 \%$ (16/ 47): $8.8 \%$ in PI-RADS category $3 \mathrm{~A}$ (3/34), $100 \%$ in PIRADS category 3B (10/10), and $100 \%$ in PI-RADS category $4(3 / 3)$.

\section{Analysis of inter-reader agreement and diagnostic performance for PI-RADS v2.1}

Regarding inter-reader agreement for malignancy detection, the kappa value was 0.949 for PI-RADS v2.1 (indicating almost perfect agreement) and 0.933 for PI-RADS v2.1-B (indicating almost perfect agreement). In PIRADS v2.1, there was disagreement for four patients; in PI-RADS v2.1-B, there was disagreement for five.

In comparing the diagnostic performance for TZPC detection between PI-RADS v2.1 and PI-RADS v2.1-B, there was no significant difference in diagnostic sensitivity for either reader $(100 \%$ vs. $97.8 \%, p=.16$; and 

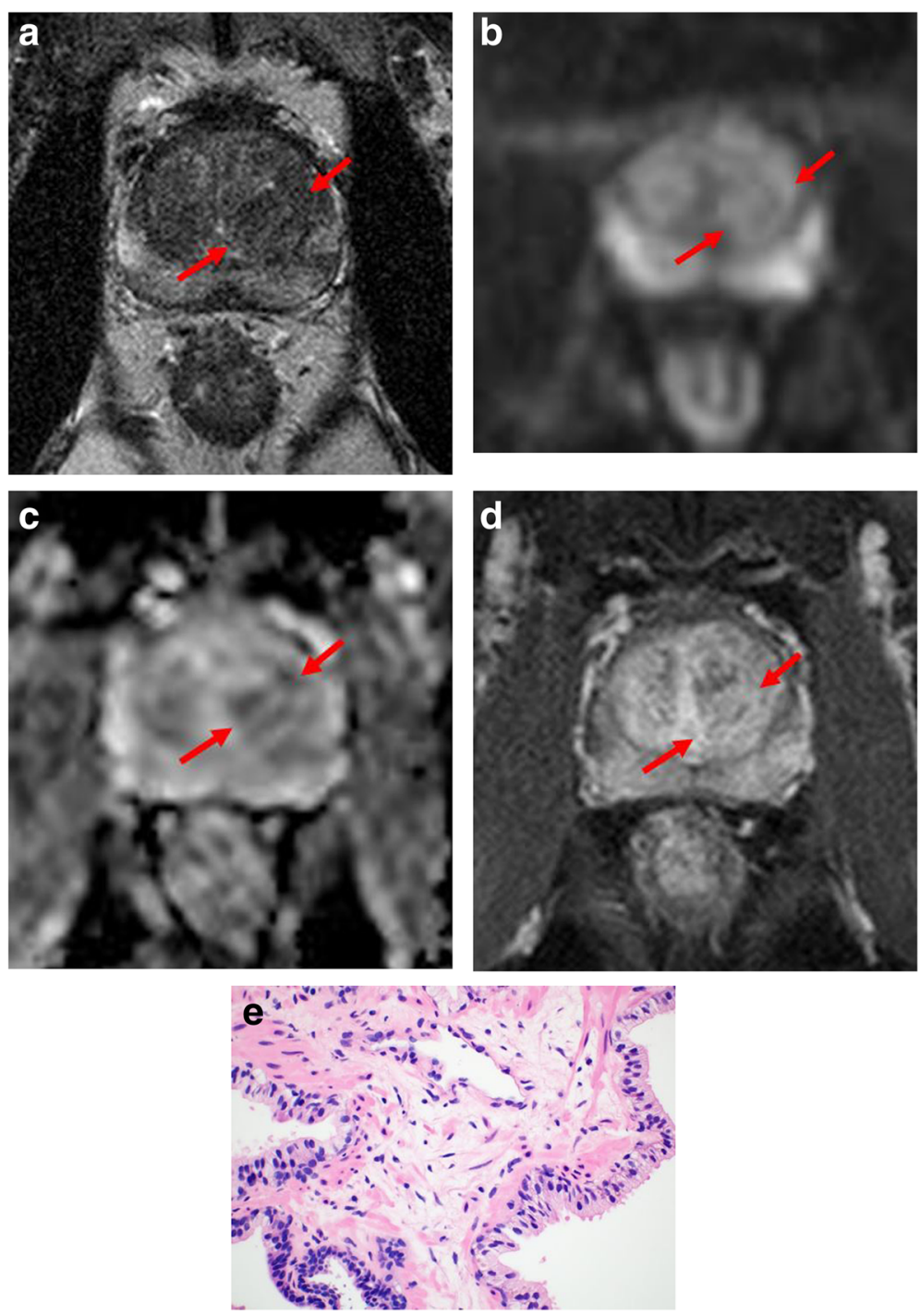

Fig. 4 Multiparametric magnetic resonance image of a 61-year-old man with a left transition-zone nodule (serum prostate-specific antigen 5.318 $\mathrm{ng} / \mathrm{ml}$, apparent diffusion coefficient [ADC] 0.868). An axial T2-weighted image (T2WI) (a) and dynamic contrast-enhanced image (b) indicate an approximately $1 \mathrm{~cm}$, heterogeneous, obscured, marginated nodule with minimal enhancement in the left transition zone (arrows), resulting in a T2WI score of 3. Diffusion-weighted imaging (DWI) $(b=1000)(\mathbf{c})$ demonstrated subtle iso-signal intensity at the corresponding nodule with low signal intensity on an ADC map (d), resulting in a DWI score of 3. This lesion was categorized as 3 according to PI-RADS V2.1 but as 3A according to PI-RADS V2.1-B. Transrectal ultrasound-guided prostate biopsy was performed. Pathology confirmed a benign hyperplastic nodule, composed of hyperplastic glands with papillary infoldings and lined by double-layered inner columnar and outer cuboidal epithelium (e)

$100 \%$ vs. $96.7 \%, p=.08$ ) (Table 4 ). The diagnostic specificity of v2.1-B was significantly higher for both reader $1(38.1 \%$ vs. $93.7, p<.001)$ and reader 2 $(17.0 \%$ vs. $90.5 \%, p<.001)$. The positive predictive value differed significantly between v2.1 and v2.1-B for both readers $(70.0 \%$ vs. $95.7 \%, p<.001$; and $70.0 \%$ vs. $93.6 \%, p<.001)$. The negative predictive value did not differ significantly for either reader $(100 \%$ vs. $96.7 \%, p=.37$; and $100 \%$ vs. $95.0 \%, p=$ $.52)$. The area under the curve tended to be higher in v2.1-B for both readers (0.933 vs. 0.972 ; and 0.926 vs. $0.960)$.
After reaching a consensus in case of disagreement, the diagnostic performances for TZPC between PIRADS v2.1 and PI-RADS v2.1-B were also evaluated (Table 5). Diagnostic sensitivity did not differ significantly between v2.1 and v2.1-B (100\% vs. $94.1 \%, p=$ $.15)$, but diagnostic specificity did differ significantly $(22.7 \%$ vs. $97.7 \%, p<.001)$. The positive predictive value was significantly higher in v2.1 $(50.0 \%$ vs. $97.0 \%, p<$ .001 ), but the negative predictive value showed no significant difference between the two versions $(100 \%$ vs. $95.6 \%, p=.497)$. The area under the curve tended to be higher in v2.1-B (0.834 vs. 0.970). 

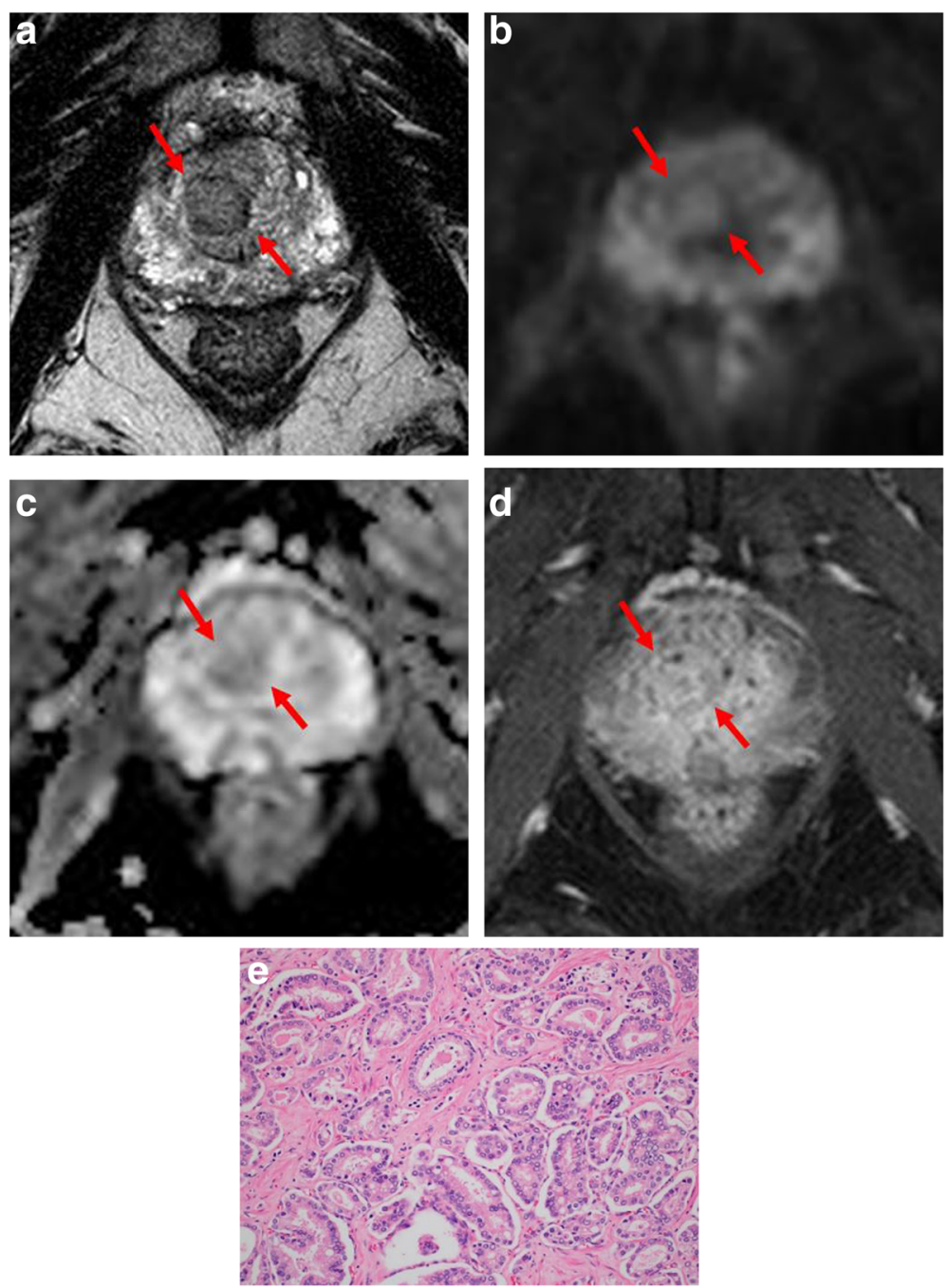

Fig. 5 Multiparametric magnetic resonance image of a 69-year-old patient who underwent radical prostatectomy (serum prostate-specific antigen $8.3 \mathrm{ng} / \mathrm{ml}$, apparent diffusion coefficient [ADC 1.140). An axial T2-weighted image (T2WI) (a) and dynamic contrast-enhanced image (b) indicate an approximately $1.3 \mathrm{~cm}$, heterogeneous, obscured, marginated nodule with mild enhancement in the right transition zone (arrows), resulting in a T2WI score of 3. Diffusion-weighted imaging (DWI) $(b=1000)(\mathbf{c})$ demonstrated subtle iso-signal intensity at the corresponding nodule with low signal intensity on an ADC map (d), resulting in a DWI score of 3. This lesion was categorized as 3 according to PI-RADS v2.1 but as 3A according to PI-RADS V2.1-B. The patient underwent radical prostatectomy, which confirmed transition-zone cancer with a Gleason score of $7(4+3)$. The procedure revealed crowded irregular glands with at least a wisp of stroma between each gland (e). This was a false-negative case first categorized with a T2 score of 3 and DWI score of 3. The final category was determined to be 3 based on v2.1 and 3A based on v2.1-B, which is pathologically confirmed malignancy

Table 4 Comparison of diagnostic performance for transition zone prostate cancer detection between PI-RADS V2.1 and PI-RADS v2.1-B

\begin{tabular}{|c|c|c|c|c|c|c|}
\hline & \multicolumn{3}{|l|}{ Reader 1} & \multicolumn{3}{|l|}{ Reader 2} \\
\hline & \multicolumn{2}{|l|}{ PI-RADS } & \multirow{2}{*}{$\begin{array}{l}P \\
\text { value }\end{array}$} & \multicolumn{2}{|l|}{ PI-RADS } & \multirow{2}{*}{$\begin{array}{l}P \\
\text { value }\end{array}$} \\
\hline & v 2.1 & v 2.1-B & & v 2.1 & v 2.1-B & \\
\hline Sensitivity & $100(91 / 91)$ & $97.8(89 / 91)$ & 0.155 & $100(91 / 91)$ & $96.7(88 / 91)$ & 0.081 \\
\hline Specificity & $38.1(24 / 63)$ & $93.7(59 / 63)$ & $<0.000$ & $17.0(8 / 47)$ & $90.5(57 / 63)$ & $<0.000$ \\
\hline Positive predictive value & $70.0(91 / 130)$ & $95.7(89 / 93)$ & $<0.000$ & $70.0(91 / 130)$ & $93.6(88 / 94)$ & $<0.000$ \\
\hline Negative predictive value & $100(24 / 24)$ & $96.7(59 / 61)$ & 0.369 & $100(8 / 8)$ & $95.0(57 / 60)$ & 0.518 \\
\hline AUC & $0.933(0.896-0.971)$ & $0.972(0.946-0.998)$ & & $0.926(0.896-0.965)$ & $0.960(0.929-0.991)$ & \\
\hline
\end{tabular}

AUC area under the receiver operating characteristic curve 
Table 5 Diagnostic performance of transition zone prostate cancer detection between PI-RADS V2.1 and PI-RADS V2.1-B

\begin{tabular}{|c|c|c|c|}
\hline & \multicolumn{2}{|l|}{ PI-RADS } & \multirow{2}{*}{$\begin{array}{l}P \\
\text { value }\end{array}$} \\
\hline & v 2.1 & v 2.1-B & \\
\hline Sensitivity & $100(34 / 34)$ & $94.1(32 / 34)$ & 0.151 \\
\hline Specificity & $22.7(10 / 44)$ & $97.7(43 / 44)$ & $<0.000$ \\
\hline Positive predictive value & $50.0(34 / 68)$ & $97.0(32 / 33)$ & $<0.000$ \\
\hline Negative predictive value & $100(10 / 10)$ & $95.6(43 / 45)$ & 0.497 \\
\hline$A \cup C$ & $0.834(0.742-0.925)$ & $0.970(0.000-1.000)$ & \\
\hline
\end{tabular}

\section{Discussion}

The PI-RADS v2.1 definition of clinically significant cancer is intended to standardize reporting of mpMRI exams and correlate with pathology for clinical and research applications; based on the current uses and capabilities of mpMRI and MRI-targeted procedures, the definition uses GS $>7$ (including $3+4$ with prominent but not predominant Gleason 4 component), volume $>$ $0.5 \mathrm{cc}$, and/or extraprostatic extension. The PI-RADS v2.1 assessment uses a 5-point scale based on the probability of clinically significant cancer, using mpMRI findings on T2W, DWI, and DCE-MRI.

In this study, all lesions were re-analyzed using the modified PI-RADS v2.1-B: our new PI-RADS subclassification. We subclassified PI-RADS category $3 \mathrm{TZ}$ lesions as PI-RADS category 3B (biopsy needed; T2WI score 3 with DWI score 4) or PI-RADS category 3A (no biopsy needed; T2WI score 3 with DWI score 1, 2, or 3). Figure 6 reveals the appropriate examples of this study.
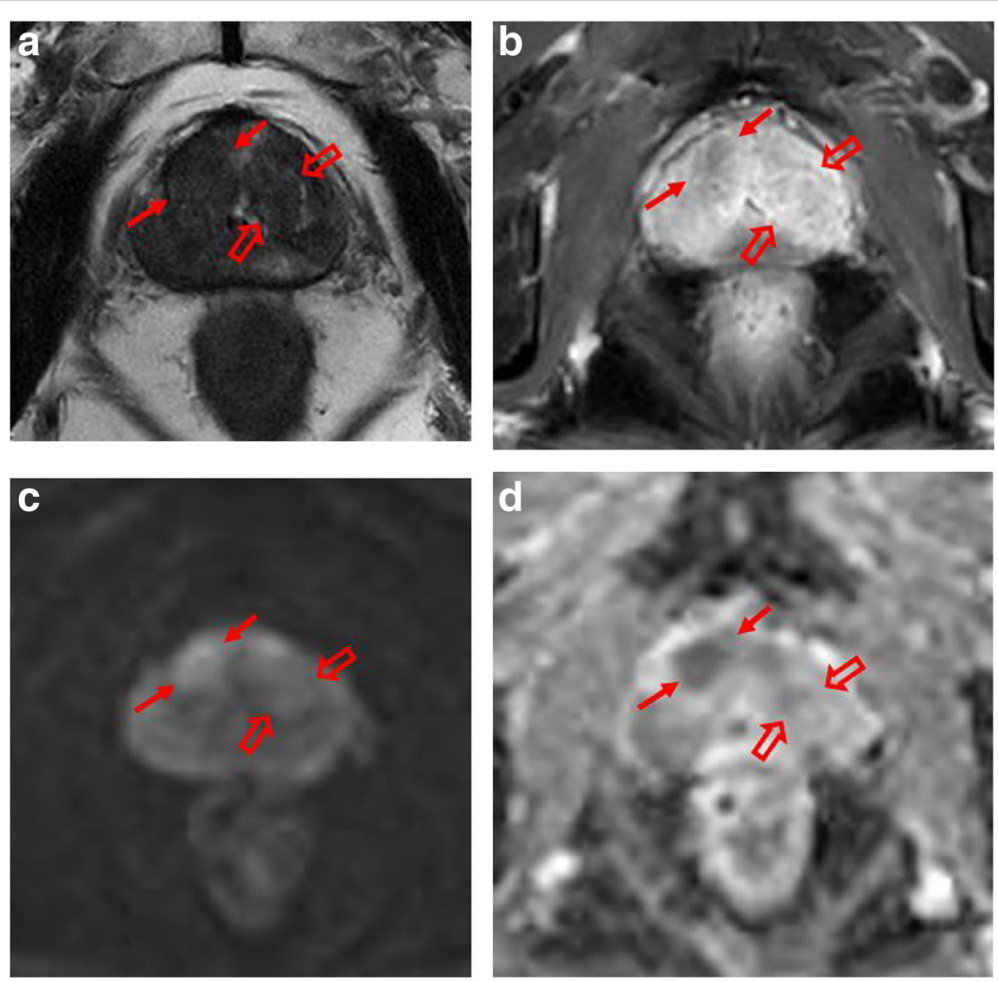

Fig. 6 Multiparametric magnetic resonance image of a 81-year-old patient with serum PSA $15.9 \mathrm{ng} / \mathrm{ml}$. An axial T2-weighted image (T2WI) and dynamic contrast-enhanced image $(\mathbf{a}, \mathbf{b})$ indicate an approximately $1.3 \mathrm{~cm}$, obscured marginated, heterogeneous nodule with minimal enhancement in the right transition zone (arrows), resulting in a T2WI score of 3. The other 9-mm-sized encapsulated, homogeneous nodule was detected in the left transition zone (opened arrows), revealing T2WI score of 2. Diffusion-weighted imaging (DWI) $(b=1000)$ and ADC map (c, d) demonstrated focal marked diffusion restriction at the corresponding right transition zone nodule (arrows) with low signal intensity on an ADC map (DWI score 4, with ADC value of 0.620 ), and left transition zone nodule revealed no diffusion restriction (DWI score 1, with ADC value of 1.040). These lesions were each categorized as category $3 B$ (right transition zone) and 2 (left transition zone), according to PI-RADS V2.1-B, and we recommended biopsy for right transition zone nodule. These lesions were confirmed right transition-zone cancer with a Gleason score of $7(3+$ 4), and benign hyperplastic nodule in left transition zone, each 
We compared clinicopathologic characteristics and ADC values between T2WI score 3 lesions of TZPC and evaluated the diagnostic performances and inter-reader agreements of the modified PI-RADS v2.1-B and PIRADS v2.1. We found the malignancy rate of T2WI score 3 lesions of TZPC differed among the three groups: $8.8 \%$ in PI-RADS category 3A, $100 \%$ in PI-RADS category 3B, and $100 \%$ in PI-RADS category 4 . The average ADC value was significantly different between the PI-RADS category $3 \mathrm{~A}$ and PI-RADS category $3 \mathrm{~B}$ groups, but no difference was found between the PI-RADS category $3 \mathrm{~B}$ and PIRADS category 4 groups. This suggests that the lesions included in PI-RADS category 3B share many characteristics with those in PI-RADS category 4, and biopsy may be needed due to the high possibility of malignancy.

In PI-RADS v2, a score of 3 or more has been used as a threshold to determine the need for biopsy, but the detection rate among PI-RADS category 3 lesions of TZ revealed considerable variation in several studies, ranging from 3.8 to $33.1 \%$ [17-19]. In addition, one study suggested that, according to PI-RADS v2, the prostate lesions characterized on 3T mpMRI as PI-RADS category 3 have a low likelihood of clinically significant prostate cancer [20]. For this reason, some prior studies have suggested the optimal cutoff for biopsy requirement in TZ lesions is a score of 4 or more, as it has high specificity $[21,22]$. However, due to the possibility of malignancy in TZ lesions with T2WI score 3 and DWI score 4, subcategorization of these lesions should be considered. The need for subcategorization is further supported by the high malignancy rate among these lesions in this study.

Several previous study presented variability of interreader agreement and diagnostic performance of PIRADS v2.1 in detecting prostate cancer, especially TZPC $[23,24]$. One study revealed inter-reader agreement improved in PZPC using PI-RADS v2.1 but not TZPC, and suggested areas where additional modification of PIRADS v2.1 could further improve inter-reader agreement and diagnostic performance [25]. In this study, by subcategorization of T2WI score 3 lesion of TZ, the level of inter-reader agreement in malignancy detection between the two readers had never lagged behind; almost perfect agreement for PI-RADS v2.1-B ( $\kappa=0.933)$ in addition to PI-RADS v2.1 ( $\mathrm{K}=0.949)$. After comparing the diagnostic performances of the two readers for TZPC detection using PI-RADS v2.1 and PI-RADS v2.1$\mathrm{B}$, we found that PI-RADS v2.1-B had a higher specificity and positive predictive value for both readers; these differences were statistically significant. Furthermore, after consensus in the case of disagreement, PI-RADS v2.1-B had significantly higher specificity and positive predictive value. Through this, PI-RADS v2.1-B may contribute to the detection of TZPC more accurately.
There were several limitations to this study. First, this was a retrospective study performed at a single center, which may have resulted in selection bias. Second, the number of lesions included in our study was low, leading to underpowered statistical analysis. This study is a work for testing a subcategorization of PI-RADS category 3 TZ lesions. Thus, larger multi-center studies including large number of patients are needed to validate our findings and modify of PI-RADS v2.1. Third, this study assessed diagnostic performance only for TZ lesions, and prostate cancer occurs more frequently in the PZ. However, we felt it necessary to focus on TZ lesions due to the ambiguity of T2WI scoring of the TZ. Finally, analysis of previously detected lesions may have impacted inter-reader agreement and diagnostic performance.

\section{Conclusions}

This study showed significantly higher specificity and positive predictive value with PI-RADS v2.1-B than with PI-RADS v2.1. PI-RADS category 3B lesions, which were upgraded based on DWI score, tended to have greater potential for malignancy than PI-RADS category 3A lesions. This indicates that subcategorization of PI-RADS category $3 \mathrm{TZ}$ lesions may contribute to accurate evaluation and effective clinical management. This study is a test of subcategorization of PI-RADS category 3 TZ lesions, so multicenter studies are needed to validate this study and modify of PI-RADS v2.1.

\section{Abbreviations}

ADC: Apparent diffusion coefficient; DCE: Dynamic contrast enhanced; DWI: Diffusion-weighted imaging; FOV: field of view; GS: Gleason score; mpMRI: Multiparametric magnetic resonance imaging; NEX: Number of excitations; PI-RADS: Prostate Imaging and Reporting Data System; PI-RADS V2.1: PI-RADS Version 2.1; PZ: Peripheral zone; TIC: Time-signal intensity curve; TR/TE/FA: Repetition time/echo time/fractional anisotropy; TSE: T2-weighted turbo spin-echo; TZ: Transition zone; TZPC: TZ prostate cancer

Acknowledgements

Not applicable.

Availability of data and material

Not applicable.

\section{Authors' contributions}

$\mathrm{JH}$ interpreted the patients' MR image, and was a major contributor in writing the manuscript. YJ and JY reviewed and analyzed the patients' data regarding clinicopathologic features and operation history. $\mathrm{CM}$ and $\mathrm{DH}$ rearranged and reviewed the data and ruled out date that doesn't meet the criteria we set. MS performed the statistics with the collected data. KW interpreted the patients' MR image, and in charge of all the progress. All authors read and approved the final manuscript.

\section{Funding \\ Not applicable.}

\section{Declarations}

Ethics approval and consent to participate Our institutional review board (Konyang University Hospital Institutional Review Board) approved this study, and the need to obtain informed patient 
consent was waived due to the retrospective nature of the study. Reference number is not applicable.

\section{Consent for publication}

Not applicable.

\section{Competing interests}

The authors declare that they have no competing interests.

\section{Author details}

'Department of Radiology, Konyang University Hospital, Konyang Univ. Hospital 158, Gwanjeodong-ro, Seo-gu, Daejeon, Korea. ${ }^{2}$ Department of Preventive Medicine, Konyang University Hospital, Konyang Univ. Hospital 158, Gwanjeodong-ro, Seo-gu, Daejeon, Korea.

Received: 31 March 2021 Accepted: 24 June 2021

Published online: 03 August 2021

\section{References}

1. Torre LA, Siegel RL, Ward EM, Jemal A (2016) Global cancer incidence and mortality rates and trends-an update. Cancer Epidemiol Biomark Prev 25(1): 16-27. https://doi.org/10.1158/1055-9965.EPI-15-0578

2. Tore LA, Bray F, Siegel RL, Ferlay J, Lortet-Tieulent J, Jemal A (2015) Global cancer statistics, 2012. CA Cancer J Clin 65:87-108

3. Kimura T, Egawa S (2018) Epidemiology of prostate cancer in Asian countries. Int J Urol 25(6):524-531. https://doi.org/10.1111/iju.13593

4. Choi E, Lee S, Nhung BC, Suh M, Park B, Jun JK, Choi KS (2017) Cancer mortality-to-incidence ratio as an indicator of cancer management outcomes in Organization for Economic Cooperation and Development countries. Epidemiol Health 39:e2017006. https://doi.org/10.4178/epih.e201 7006

5. McNeal JE, Redwine EA, Freiha RS, Stamey TA (1988) Zonal distribution of prostatic adenocarcinoma. Correlation with histologic pattern and direction of spread. Am J Surg Pathol 12(12):897-906. https://doi.org/10.1097/000004 78-198812000-00001

6. Patel P, Wang S, Siddiqui MM (2019) The use of multiparametric magnetic resonance imaging (mpMRI) in the detection, evaluation, and surveillance of clinically significant prostate cancer (csPCa). Curr Urol Rep 20:60

7. EL-Adalany M, EL-Razek A, EL-Diasty T, EL-Hendy A, EL-Metwally D (2021) Comparison between biparametric and multiparametric MR imaging of Prostate Imaging Reporting and Data System Version 2.1 in detection of prostate cancer. Egypt J Radiol Nucl Med 52:68

8. Zhen L, Liu X, Yegang C et al (2019) Accuracy of multiparametric magnetic resonance imaging for diagnosing prostate cancer: a systematic review and meta-analysis. BMC Cancer 19:1244

9. Rosenkrantz AB, Ginocchio LA, Cornfeld D, Froemming AT, Gupta RT, Turkbey B, Westphalen AC, Babb JS, Margolis DJ (2016) Interobserver reproducibility of the PI-RADS Version 2 lexicon: a multicenter study of six experienced prostate radiologist. Radiology 280(3):793-804. https://doi.org/1 $0.1148 /$ radiol.2016152542

10. Borofsky W, George AK, Gaur S et al (2018) What are we missing? Falsenegative cancers at multiparametric MR imaging of the prostate. Radiology 286(1):186-195. https://doi.org/10.1148/radiol.2017152877

11. Chesnais AL, Niaf E, Bratan F, Mège-Lechevallier F, Roche S, Rabilloud M, Colombel M, Rouvière O (2013) Differentiation of transitional zone prostate cancer from benign hyperplasia nodules: evaluation of discriminant criteria at multiparametric MRI. Clin Radiol 68(6):e323-e330. https://doi.org/10.1016/ j.crad.2013.01.018

12. Tamada T, Kido A, Takeuchi M, Yamamoto A, Miyaji Y, Kanomata N, Sone T (2019) Comparison of PI-RADS version 2 and PI-RADS version 2.1 for the detection of transition zone prostate cancer. Eur J Radiol 121:108704. https://doi.org/10.1016/j.ejrad.2019.108704

13. Byun J, Park KJ, Kim MH, Kim JK (2020) Direct comparison of PI-RADS Version 2 and 2.1 in transition zone lesions for detection of prostate cancer: preliminary experience. J Magn Reson Imaging 52(2):577-586. https://doi. org/10.1002/jmri.27080

14. Ahmed HM, Ebeed AE, Hamdy A, El-Ghar MA, Razek A (2021) Interobserver agreement of Prostate Imaging-Reporting and Data System (PI-RADS-V2). Egypt J Radiol Nucl Med 52(1):5. https://doi.org/10.1186/s43055-020-00378W
15. Moreira ASL, De Visschere P, Van Praet C, Villeirs G (2020) How does PIRADS v2.1 impact patient classification? A head-to-head comparison between PI-RADS v2.0 and v2.1. Acta Radiol 62(6):839-847. https://doi.org/1 $0.1177 / 0284185120941831$

16. Epstein Jl, Egevad I, Amin MB et al (2016) The 2014 International Society of Urological Pathology (ISUP) Consensus Conference on Gleason grading of prostatic carcinoma: definition of grading patterns and proposal for a new grading system. Am J Surg Pathol 40(2):244-252. https://doi.org/10.1097/PA S.0000000000000530

17. Felker ER, Raman SS, Margolis DJ, Lu DSK, Shaheen N, Natarajan S, Sharma D, Huang J, Dorey F, Marks LS (2017) Risk stratification among men with prostate imaging reporting and data system version 2 category 3 transition zone lesions: is biopsy always necessary? AJR Am J Roentgenol 209(6):12721277. https://doi.org/10.2214/AJR.17.18008

18. Thai JN, Narayanan HA, George AK, Siddiqui MM, Shah P, Mertan FV, Merino MJ, Pinto PA, Choyke PL, Wood BJ, Turkbey B (2018) Validation of PI-RADS version 2 in transition zone lesions for the detection of prostate cancer. Radiology 288(2):485-491. https://doi.org/10.1148/radiol.2018170425

19. Greer MD, Shih JH, Lay $N$ et al (2017) Validation of the dominant sequence paradigm and role of dynamic contrast-enhanced imaging in PI-RADS version 2. Radiology 27:161316

20. Liddell $H$, Jyoti $R$, Haxhimolla HZ (2015) mp-MRI prostate characterized PIRA DS 3 lesions are associated with a low risk of clinically significant prostate cancer: a retrospective review of 92 biopsied PIRADS 3 lesions. Curr Urol 8(2):96-100. https://doi.org/10.1159/000365697

21. Wang X, Bao J, Ping X, Hu C, Hou J, Dong F, Guo L (2018) The diagnostic value of PI-RADS V1 and V2 using multiparametric MRI in transition zone prostate clinical cancer. Oncol Lett 16(3):3201-3206. https://doi.org/10.3892/ ol.2018.9038

22. Feng ZY, Wang L, Min XD, Wang SG, Wang GP, Cai J (2016) Prostate cancer detection with multiparametric magnetic resonance imaging: prostate imaging reporting and data system version 1 versus version 2. Chin Med J 129(20):2451-2459. https://doi.org/10.4103/0366-6999.191771

23. Wang Z, Zhao W, Shen J, Jiang Z, Yang S, Tan S, Zhang Y (2020) PI-RADS version 2.1 scoring system is superior in detecting transition zone prostate cancer: a diagnostic study. Abdom Radiol (NY) 45(12):4142-4149. https://doi. org/10.1007/s00261-020-02724-y

24. Xu L, Zhang G, Zhang D, Zhang X, Bai X, Yan W, Zhou Y, Zhou Z, Xiao Y, Jin $Z$, Sun $H$ (2020) Comparison of PI-RADS version 2.1 and PI-RADS version 2 regarding interreader variability and diagnostic accuracy for transition zone prostate cancer. Abdom Radiol (NY) 45(12):4133-4141. https://doi.org/10.1 007/s00261-020-02738-6

25. Bhayana R, O'Shea A, Anderson MA, Bradley WR, Gottumukkala R, Mojtahed A, Pierce TT, Harisinghani M (2020) PI-RADS version 2 and 2.1: Interobserver agreement and diagnostic performance in peripheral and transition zone lesions among six radiologists. AJR Am J Roentgenol 217(1):141-151. https://doi.org/10.2214/AJR.20.24199

\section{Publisher's Note}

Springer Nature remains neutral with regard to jurisdictional claims in published maps and institutional affiliations.

\section{Submit your manuscript to a SpringerOpen ${ }^{\odot}$ journal and benefit from:}

- Convenient online submission

- Rigorous peer review

- Open access: articles freely available online

- High visibility within the field

- Retaining the copyright to your article

Submit your next manuscript at $>$ springeropen.com 